\title{
ASPECTOS QUÍMICOS E FARMACOLÓGICOS DE Byrsonima (MALPIGHIACEAE)
}

\section{Fernanda Guilhon-Simplicio ${ }^{\#}$}

Centro Universitário Nilton Lins, Av. Prof. Nilton Lins, 3259, 69058-030 Manaus - AM, Brasil

Maria de Meneses Pereira*

Faculdade de Ciências Farmacêuticas, Universidade Federal do Amazonas, Rua Alexandre Amorim, 330, 69010-300 Manaus AM, Brasil

Recebido em 13/7/10; aceito em 24/12/10; publicado na web em 15/4/11

\begin{abstract}
CHEMICAL AND PHARMACOLOGICAL ASPECTS OF Byrsonima (Malpighiaceae). Species of the Byrsonima genus are widely distributed around the neotropical zone, being frequently used in folk medicine to treat gastrointestinal, respiratory and skin diseases. This article briefly reviews the ethnopharmacology, pharmacology and phytochemistry of the Byrsonima genus. Eighty three compounds isolated from different species are reported, most of them being flavonoids or triperpenes. The pharmacological studies carried out with the extracts from these plants emphasize on the antimicrobial activity, however other activities have also been investigated leading to promising results. The data presented in this work strongly supports the view that plants of Byrsonima genus have potential therapeutic action.
\end{abstract}

Keywords: Malpighiaceae; Byrsonima; review.

\section{INTRODUÇÃO}

Malpighiaceae é uma família de árvores, arbustos, subarbustos e lianas com aproximadamente 71 gêneros e 1250 espécies, que se distribuem em florestas tropicais, subtropicais e savanas do Velho e Novo Mundo, onde estão localizadas $85 \%$ das espécies. ${ }^{1}$ Várias espécies dessa família são utilizadas com fins medicinais pela população de países americanos, principalmente como moduladores do sistema nervoso central (gêneros Banisteriopsis, Diplopterys, Galphimia e Heteropteris) e afrodisíacos (gênero Heteropteris). ${ }^{2}$

Uma revisão acerca da constituição química dessa família registra como principais fitoconstituintes os alcaloides carbolínicos, bases indólicas simples, fenilpropanoides, flavonoides, triterpenoides e polissacarídeos em diferentes gêneros. Esse mesmo trabalho destaca o uso ritualístico-religioso de algumas espécies, que é registrado até os dias atuais, além o valor nutricional de espécies do gênero Malpighia, ricas em ácido ascórbico (vitamina C) em seus frutos, que são saborosos e bastante apreciados na culinária brasileira. ${ }^{3}$

Entretanto, o gênero mais extensivamente estudado tem sido o Byrsonima, um dos maiores da família Malpighiaceae, possuindo 150 espécies com distribuição marcadamente neotropical. ${ }^{4} \mathrm{O}$ Brasil concentra cerca de $50 \%$ das espécies, que são encontradas principalmente nas regiões Norte, Nordeste e Central, podendo também ser encontradas na região Sudeste do país, em áreas de cerrado. No Brasil, essas espécies são conhecidas popularmente como "muricis", sendo diferenciadas pela cor de suas flores e frutos, ou pelo local de ocorrência. Desse modo, recebem nomes como murici da várzea, murici da mata, murici-amarelo, entre outros. ${ }^{5}$

A madeira das espécies arbóreas é leve, sendo utilizada na construção civil para a fabricação de caibros e vigas. Algumas delas já foram bastante empregadas em curtumes e para tingir tecidos, devido à

*e-mail: maria.menesespereira@gmail.com

"Endereço atual: Faculdade de Ciências Farmacêuticas, Universidade Federal do Amazonas, Manaus - AM, Brasil grande quantidade de taninos e matéria tintorial nas cascas. Com flores vistosas, frutos que atraem aves e rápido crescimento, tais espécies têm grande potencial como plantas ornamentais e também são úteis na recuperação de áreas degradadas. ${ }^{6}$ Os frutos de $B$. crassifolia e $B$. verbascifolia são bastante apreciados para consumo in natura e utilizados na preparação de bebidas e doces em diversas regiões do país. ${ }^{7}$

Além do uso da madeira na construção civil e dos frutos como alimento, várias partes de diversas espécies têm amplo emprego popular como medicamentos. A Tabela 1 mostra alguns usos etnofarmacológicos de espécies de Byrsonima reportados na literatura.

Esse extenso uso etnofarmacológico motivou vários trabalhos acerca da constituição química e do potencial farmacológico dessas espécies, interesse que tem aumentado nos últimos anos, devidos aos resultados promissores apresentados. $\mathrm{O}$ presente artigo traz uma revisão bibliográfica dos estudos químicos e/ou farmacológicos realizados com espécies do gênero Byrsonima publicados em revistas indexadas desde 1970 até os dias atuais.

\section{ESTUDOS QUÍMICO-FARMACOLÓGICOS COM ESPÉCIES DO GÊNERO Byrsonima}

As espécies do gênero Byrsonima têm sido predominantemente investigadas quanto a sua ação contra micro-organismos, entre diversas espécies de bactérias, enterobactérias, micobactérias, protozoários e fungos implicados na etiologia de doenças gastrointestinais e de pele, com resultados positivos na maioria dos estudos. Outras atividades biológicas como espasmogênica/antiespasmogênica, imunoestimuladora, anti-inflamatória tópica, anti-hemorrágica, antiglicemiante, anti-hiperlipidêmica, antiulcerogênica, antidiarreica e antioxidante também foram investigadas em diferentes espécies. De forma geral, os resultados apresentados corroboram para a validação dos usos etnofarmacológicos (Tabela 2).

Flavonoides, destacando-se, flavanois e seus heterosídeos, flavanonas, biflavonoides, catequinas e epicatequinas (estruturas de 1 a 26), proantocianidinas (estruturas de 27 a 33), compostos 
Tabela 1. Usos etnofarmacológicos de espécies de Byrsonima

\begin{tabular}{|c|c|c|c|}
\hline Espécie & Parte utilizada & Usos etnofarmacológicos & Ref. \\
\hline B. basiloba & Folha & Antidiarreico e tratamento de úlceras gástricas & 8,9 \\
\hline B. bucidaefolia & Folha & Antiasmático, febrífugo e tratamento de infecções na pele & 10 \\
\hline B. crassa & Folha e casca do caule & $\begin{array}{l}\text { Antidiarreico, antiemético, diurético, febrífugo, tratamento doenças de pele, gastrites, } \\
\text { picadas de cobra, úlceras gástricas e pépticas }\end{array}$ & $11-20$ \\
\hline B. crassifolia & Folha, casca do caule, fruto e semente & $\begin{array}{l}\text { Antiasmático, abortivo, antidiarreico (disenteria), anti-inflamatório, antimalárico, } \\
\text { digestivo, diurético, febrífugo, laxativo, tratamento de amenorréia, bronquite, cólicas, } \\
\text { dor de dente, indigestão, infecções na pele e mucosas, leucorréia, parasitoses intestinais, } \\
\text { perda de apetite, picadas de cobra, resfriados, tosses, para expelir placenta e "apertar } \\
\text { dentes frouxos" }\end{array}$ & $21-37$ \\
\hline B. fagifolia & Folha e casca do caule & Antidiarreico, antiemético, diurético, tratamento de gastrite e úlceras gástricas e pépticas & $38-40$ \\
\hline B. intermedia & Casca do caule & Tratamento de bronquites e tosses & 41 \\
\hline B. sericea & Folha & Antidiarreico e tratamento de diabetes & 42 \\
\hline B. verbascifolia & Folha, casca do caule e casca da raiz & $\begin{array}{l}\text { Antidiarreico, antipirético, cicatrizante, laxativo, tratamento de infecções na pele e } \\
\text { mucosas e doença de Chagas }\end{array}$ & $43-46$ \\
\hline
\end{tabular}

Tabela 2. Atividades farmacológicas investigadas em espécies do gênero Byrsonima

\begin{tabular}{|c|c|c|c|c|c|c|}
\hline Espécie & Parte estudada & Atividade investigada & \multicolumn{2}{|l|}{ Resultado } & Extrato(s) & Ref. \\
\hline \multirow{6}{*}{ B. basiloba } & \multirow{6}{*}{ Folha } & Antidiarreica & \multicolumn{2}{|l|}{ Ativo } & $k, l$ & 9 \\
\hline & & Antibacteriana & \multicolumn{2}{|c|}{$\begin{array}{l}\text { Ativo (Bacillus cereus, Bacillus subtilis, Enterococcus faecalis, Proteus } \\
\text { mirabilis, Salmonella sp., Shigella sp., Staphylococcus epidermidis) }\end{array}$} & $k, l$ & 47 \\
\hline & & Antifúngica & \multicolumn{2}{|c|}{ Ativo (Candida albicans) } & & \\
\hline & & Antimicobacteriana & \multicolumn{2}{|c|}{ Pouco ativo (Mycobacterium fortuitum) } & $k$ & 48 \\
\hline & & Antimutagênica & \multicolumn{2}{|c|}{ Ativo (Salmonella typhimurium) } & $e^{k}$ & 8 \\
\hline & & Mutagênica & \multicolumn{2}{|c|}{ Inativo (Salmonella typhimurium) } & $e, \kappa$ & 0 \\
\hline \multirow{3}{*}{ B. bucidaefolia } & Casca do caule & Antiprotozoária & \multicolumn{2}{|c|}{ Ativo (forma promastigota de Leishmania mexicana) } & $k$ & 49 \\
\hline & \multirow{2}{*}{ Folha } & Antioxidante & \multirow{2}{*}{\multicolumn{2}{|c|}{ Ativo (forma promastigota de Leishmania mexicana) }} & $c, f, g, h, m$ & 10 \\
\hline & & Antiprotozoária & & & $k$ & 49 \\
\hline \multirow{5}{*}{ B. coccolobifolia } & \multirow{5}{*}{ Folha } & Antihocterian & \multicolumn{2}{|c|}{ Ativo (Bacillus cereus, Pseudomonas aeruginosa, Staphylococcus aureus) } & & \\
\hline & & 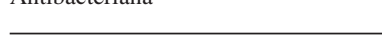 & \multicolumn{2}{|c|}{ Inativo (Escherichia coli) } & & \\
\hline & & Antifúngica & \multicolumn{2}{|c|}{ Inativo (Cladosporium sphaerospermum) } & $d$ & 50 \\
\hline & & Citotóxica & \multicolumn{2}{|c|}{ Inativo (Artemia salina) } & & \\
\hline & & Moluscicida & \multicolumn{2}{|c|}{ Ativo (Biomphalaria glabrata) } & & \\
\hline \multirow{14}{*}{ B. crassa } & Casca do caule & Antimicobacteriana & Ativo (Mycol & acterium tuberculosis) & $e, o$ & 14 \\
\hline & \multirow{13}{*}{ Folha } & Alelopática & Ativo & & $l, q, t$ & 15 \\
\hline & & \multirow{2}{*}{ Antibacteriana } & \multirow[t]{2}{*}{ Ativo } & $\begin{array}{l}\text { Bacillus cereus, Bacillus subtilis, Enterococcus faecalis, Proteus } \\
\text { mirabilis, Salmonella sp., Shigella sp., Staphylococcus epidermidis }\end{array}$ & $k, l, r, s$ & 13 \\
\hline & & & & Helicobacter pylori & $e, k$ & 16 \\
\hline & & Antifúngica & \multicolumn{2}{|c|}{ Ativo (Candida albicans) } & $k, l, r, s$ & 13 \\
\hline & & Anti-inflamatória & \multicolumn{2}{|c|}{ Ativo (inibição da produção de NO e fator de necrose tumoral $\alpha$ ) } & $p$ & 20 \\
\hline & & & Ativo $(\mathrm{Mycol}$ & acterium tuberculosis) & $e, o$ & 14,51 \\
\hline & & Antimicobacteriana & & Mycobacterium fortuitum & $e, h$ & 48 \\
\hline & & & Pouco ativo & Mycobacterium tuberculosis & $k$ & 51 \\
\hline & & Antiproliferativa & Ativo (inibiç & da proliferação de células tumorais de mamíferos) & $p$ & 20 \\
\hline & & Antiulcerogênica & Ativo & & $k, l$ & 17 \\
\hline & & Imunoestimuladora & Ativo (induç & da produção de $\mathrm{H}_{2} \mathrm{O}_{2}$ e/ou NO por macrófago) & $e, k$ & 16 \\
\hline & & $\begin{array}{l}\text { Inibidora de hemorragia causada pelo } \\
\text { veneno de Bothrops jararaca }\end{array}$ & Muito ativo & & $k, p$ & 18 \\
\hline & & Mutagênica & Ativo & & $e, k, l$ & 11 \\
\hline & & & & $\begin{array}{l}\text { Bacillus subtilis, Pseudomonas aeruginosa, Salmonella typhi, Shi- } \\
\text { gella flexineri, Staphylococcus aureus, Streptococcus pneumoniae }\end{array}$ & $f$ & 31 \\
\hline & & & Ativo & Pseudomonas aeruginosa, Staphylococcus aureus & $h, m$ & 27 \\
\hline & & & & Salmonella typhi & $g, h$ & 33 \\
\hline & & & & Streptococcus pneumoniae & $h$ & 32 \\
\hline & & & & Escherichia coli, Salmonella typhi, Shigella flexineri & $b$ & 32 \\
\hline B. crassifolia & Casca do caule & Antibacteriana & & Escherichia coli, Shigella flexineri & $g, h$ & 35 \\
\hline & & & & $\begin{array}{l}\text { Klebsiela pneumoniae, Staphylococcus epidermidis, Micrococcus } \\
\text { luteus, Escherichia coli }\end{array}$ & $f$ & 31 \\
\hline & & & Inativo & Pseudomonas aeruginosa, Staphylococcus aureus & $b$ & 27 \\
\hline & & & & Salmonella typhi & $b, h, m$ & 21 \\
\hline & & & & Streptococcus pneumoniae & $b, g$ & 32 \\
\hline & & & & Streptococcus pyogenes & $b, g, h$ & 5 \\
\hline
\end{tabular}


Tabela 2. continuação

\begin{tabular}{|c|c|c|c|c|c|c|}
\hline Espécie & Parte estudada & Atividade investigada & \multicolumn{2}{|c|}{ Resultado } & Extrato(s) & Ref. \\
\hline & \multirow{19}{*}{ Casca do caule } & Anti-inflamatória tópica & \multicolumn{2}{|c|}{ Ativo } & $a, e, k$ & 34 \\
\hline & & Antioxidante & \multicolumn{2}{|l|}{ Ativo } & $n$ & 30 \\
\hline & & \multirow{4}{*}{ Antifúngica } & \multirow{4}{*}{ Ativo } & Candida albicans & $h, j$ & 27,35 \\
\hline & & & & Cryptococcus neoformans & $h, m, l$ & 27 \\
\hline & & & & $\begin{array}{l}\text { Epidermophyton floccosum, Miscrosporum canis, Microsporum } \\
\text { gypseum, Trichophyton mentagrophytes var. algodonosa, Tricho- } \\
\text { phyton mentagrophytes var. granulare Trichophyton rubrum }\end{array}$ & $m$ & 25 \\
\hline & & & & Microsporum gypseum) & $h$ & 27 \\
\hline & & \multirow{4}{*}{ Antifúngica } & \multirow{3}{*}{ Inativo } & Aspergillus flavus & $c, h, j, m$ & 26,27 \\
\hline & & & & Candida albicans, Microsporum gypseum & $c, m$ & \multirow{2}{*}{27} \\
\hline & & & & Cryptococcus neoformans & $c$ & \\
\hline & & & \multicolumn{2}{|c|}{$\begin{array}{l}\text { Muito ativo (Epidermophyton floccosum, Microsporum canis, Trichophyton } \\
\text { rubrum) }\end{array}$} & $j$ & 26 \\
\hline & & \multirow{4}{*}{ Antiprotozoária } & \multirow{2}{*}{ Ativo } & Forma promastigota de Leishmania mexicana & $k$ & 49 \\
\hline & & & & Forma tripomastigota de Trypanossona cruzi & $m$ & 23 \\
\hline & & & \multirow{2}{*}{ Inativo } & Forma epimastigota de Trypanossoma cruzi & \multirow{2}{*}{$b, c, h, m$} & \multirow{3}{*}{23,27} \\
\hline & & & & Forma tripomastigota de Trypanossoma cruzi & & \\
\hline & & Citotóxica & Inativo $(A$ & ia salina) & $b, c, h, m$ & \\
\hline & & \multirow{2}{*}{ Enterobactericida } & \multicolumn{2}{|c|}{ Ativo (Salmonella typhi, Shigella flexneri) } & & \\
\hline & & & Inativo $(E$ & richia coli, Salmonella enteritidis, Shigella dysenteriae) & $J$ & 36 \\
\hline & & Espasmogênica & Ativo & & $h$ & 37 \\
\hline & & $\begin{array}{l}\text { Estimulante/depressora do sistema } \\
\text { nervoso central }\end{array}$ & Ativo (ef & epressor) & $m$ & 52 \\
\hline & \multirow{9}{*}{ Folha } & Antioxidante & Ativo & & $n$ & 30 \\
\hline & & & \multicolumn{2}{|c|}{ Inativo (Aspergillus flavus) } & & \\
\hline & & Antifúngica & $\begin{array}{l}\text { Pouco ati } \\
\text { rubrum) }\end{array}$ & pidermophyton floccosum, Microsporum canis, Trichophyton & $j$ & 26 \\
\hline \multirow{24}{*}{ B. crassifolia } & & & & Giardia lamblia & $k$ & 24 \\
\hline & & Antiprotozoária & Ativo & Forma promastigotade Leishmania mexicana & $k$ & 49 \\
\hline & & & & Forma tripomastigota de Trypanossoma cruzi & $b, h, m$ & \\
\hline & & Antiprotozoária & Inativo $(\mathrm{F}$ & a epimastigota de Trypanossoma cruzi) & $b, h, m$ & 23 \\
\hline & & Citotóxica & Inativo $(A$ & ia salina) & $b, h, m$ & \\
\hline & & Espasmogênica & Ativo & & $h, p$ & 22,37 \\
\hline & & & Inativo $(A$ & gillus flavus) & & \\
\hline & Flor & Antifúngica & $\begin{array}{l}\text { Muito ati } \\
\text { rubrum) }\end{array}$ & pidermophyton floccosum, Microsporum canis, Trichophyton & $j$ & 26 \\
\hline & & Antioxidante & Ativo & & $b, e, n$ & 30,53 \\
\hline & & & Inativo $(A$ & gillus flavus) & & \\
\hline & Fruto & Antifúngica & $\begin{array}{l}\text { Muito ati } \\
\text { rubrum) }\end{array}$ & pidermophyton floccosum, Microsporum canis, Trichophyton & $j$ & 26 \\
\hline & & Anti-hiperglicêmica & Ativo & & & \\
\hline & & Anti-hiperlipidêmica & Ativo & & $b, e$ & 53 \\
\hline & & Tóxica & Inativo (r & & & \\
\hline & & & Ativo & $\begin{array}{l}\text { Bacillus subtilis, Escherichia coli, Klebsiella pneumoniae, Mi- } \\
\text { crococcus luteus, Pseudomonas aeruginosa, Salmonella typhi, } \\
\text { Shigella flexineri, Staphylococcus aureus, Staphylococcus epi- } \\
\text { dermidis, Streptococcus pneumoniae }\end{array}$ & $f$ & \\
\hline & Raiz & Antibacteriana & . & $\begin{array}{l}\text { Escherichia coli, Klebsiella pneumoniae, Salmonella typhi, } \\
\text { Staphylococcus aureus }\end{array}$ & $k$ & 31 \\
\hline & Kalz & & $\begin{array}{l}\text { Inativo }(B \\
\text { Shigella }\end{array}$ & $\begin{array}{l}\text { Ius subtilis, Micrococcus luteus, Pseudomonas aeruginosa, } \\
\text { eri, Staphylococcus epidermidis, Streptococcus pneumoniae }\end{array}$ & $k$ & \\
\hline & & & Inativo $(A$ & gillus flavus) & & \\
\hline & & Antifúngica & Pouco ati & pidermophyton floccosum, Microsporum canis) & $j$ & 26 \\
\hline & & & Muito ati & richophyton rubrum) & & \\
\hline & & Antioxidante & Ativo & & & \\
\hline & & Anti-hiperglicêmica & Ativo & & & \\
\hline & Semente & Anti-hiperlipidêmica & Ativo & & $b, e$ & 53 \\
\hline & & Tóxica & Inativo $(\mathrm{r}$ & & & \\
\hline & & & Pouco ati & scherichia coli, Helicobacter pylori, Staphylococcus aureus) & $k, r, s$ & 38 \\
\hline B. fagifolia & Folha & Antibacteriana & $\begin{array}{l}\text { Ativo }(B a \\
\text { mirabilis }\end{array}$ & $\begin{array}{l}\text { s cereus, Bacillus subtilis, Enterococcus faecalis, Proteus } \\
\text { nonella } \text { sp., Shigella } \text { sp., Staphylococcus epidermidis) }\end{array}$ & $k, l$ & 47 \\
\hline & & Antidiarreica & Ativo & & $k$ & 38 \\
\hline & & Antifúngica & Ativo $(C c$ & (a albicans) & $k, l$ & 37 \\
\hline
\end{tabular}


Tabela 2. continuação

\begin{tabular}{|c|c|c|c|c|c|c|}
\hline Espécie & Parte estudada & Atividade investigada & \multicolumn{2}{|c|}{ Resultado } & Extrato(s) & Ref. \\
\hline \multirow{5}{*}{ B. fagifolia } & \multirow{5}{*}{ Folha } & \multirow{2}{*}{ Antimicobacteriana } & \multicolumn{2}{|c|}{ Pouco ativo (Mycobacterium fortuitum) } & $e, h$ & 48 \\
\hline & & & \multicolumn{2}{|c|}{ Ativo (Mycobacterium tuberculosis) } & $e, k, l, o$ & 39 \\
\hline & & Antioxidante & \multicolumn{2}{|c|}{ Ativo } & $k$ & \multirow{3}{*}{38} \\
\hline & & Antiulcerogênica & \multicolumn{2}{|c|}{ Ativo (ensaio do etanol/mecanismo antioxidante) } & $k$ & \\
\hline & & Mutagênica & \multicolumn{2}{|c|}{ Inativo } & $k, r, s$ & \\
\hline \multirow{2}{*}{ B. gardneriana } & \multirow{2}{*}{ Partes aéreas } & Antioxidante & \multicolumn{2}{|l|}{ Ativo } & $k$ & \multirow{2}{*}{54} \\
\hline & & Citotóxica & \multicolumn{2}{|c|}{ Inativo (Artemia salina) } & $k$ & \\
\hline \multirow{11}{*}{ B. intermedia } & \multirow{11}{*}{ Folha } & \multirow{3}{*}{ Antibacteriana } & \multirow[b]{2}{*}{ Ativo } & Bacillus cereus, Psendomonas aeruginosa, Staphylococcus aureus & $m$ & 50 \\
\hline & & & & $\begin{array}{l}\text { Bacillus cereus, Bacillus subtilis, Enterococcus faecalis, Proteus } \\
\text { mirabilis, Salmonella sp., Shigella sp., Staphylococcus epidermidis }\end{array}$ & $k, l$ & 47 \\
\hline & & & Inativo & richia coli) & $m$ & 50 \\
\hline & & & Ativo $(C$ & (a albicans) & $k, l$ & 47 \\
\hline & & Antifúngica & Inativo & sporium sphaerospermum) & $k, m$ & \\
\hline & & Citotóxica & Inativo & ia salina) & $k, m$ & 50 \\
\hline & & Genotóxica & Inativo & & $e, k, l$ & 55 \\
\hline & & & Ativo $(B$ & alaria glabrata) & $k$ & 50 \\
\hline & & Moluscicida & Inativo & Shalaria glabrata) & $m$ & 50 \\
\hline & & & Ativo $(S$ & ella typhimurium) & $k$ & \\
\hline & & Mutagênica & Inativo & nella typhimurium) & $e, l$ & 55 \\
\hline B. japurensis & Casca do caule & Antimicobacteriana & Pouco a & Aycobacterium tuberculosis) & $c$ & 56 \\
\hline & & $\begin{array}{l}\text { Estimulante/depressora do sistema } \\
\text { cardiovascular e cardiorrespiratório }\end{array}$ & Ativo (e & lepressor sobre a pressão arterial e ventilação do gato) & & \\
\hline & & $\begin{array}{l}\text { Estimulante/depressora sobre órgãos } \\
\text { isolados }\end{array}$ & $\begin{array}{l}\text { Ativo (e } \\
\text { sobre du }\end{array}$ & $\begin{array}{l}\text { stimulante sobre coração de sapo e útero de rata, tonificante } \\
\text { de coelho e inibitório sobre íleo de cobaia) }\end{array}$ & $h, m$ & 57 \\
\hline & Casca do caule & $\begin{array}{l}\text { Estimulante/depressora sobre órgãos } \\
\text { isolados }\end{array}$ & $\begin{array}{l}\text { Inativo } \\
\text { duodeno }\end{array}$ & $\begin{array}{l}\text { feito sobre a motilidade do músculo reto abdominal do sapo e do } \\
\text { elho) }\end{array}$ & & \\
\hline B. sericea & & Moluscicida & Ativo $(b$ & ialaria straminea) & $h, m$ & 58 \\
\hline & & Tóxica & Ativo (c & dongos e peixes) & $h, m$ & 57 \\
\hline & Casca da raiz & Moluscicida & Ativo $(B$ & ialaria straminea) & $h, m$ & 58 \\
\hline & & Antioxidante & Ativo & & $h$ & 42 \\
\hline & Folha & Moluscicida & Ativo $(B$ & laria straminea) & & \\
\hline & Fruto & Moluscicida & Inativo & halaria straminea) & $h, m$ & 58 \\
\hline & Madeira & Moluscicida & Ativo $(B$ & ialaria straminea) & & \\
\hline & & & Ativo $(B$ & s subtilis, Staphylococcus aureus, Streptococcus faecalis, ) & & \\
\hline & & Antibacteriana & $\begin{array}{l}\text { Inativo } \\
\text { Salmone }\end{array}$ & $\begin{array}{l}\text { richia coli, Klebsiella pneumoniae, Pseudomonas aeruginosa } \\
\text { himurium) }\end{array}$ & & \\
\hline & & Antifúngica & Inativo & da albicans) & $k$ & 44 \\
\hline & Casca da raiz & Antimicobacteriana & Ativo $(\Lambda$ & acterium phlei) & & \\
\hline & & & Ativo (v & a herpes simples) & & \\
\hline & & Antiviral & Inativo & da poliomielite) & & \\
\hline & & Estimulante de fagocitose & Ativo & & 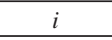 & 45 \\
\hline & & & Ativo $(B$ & scereus, Psedudomonas aeruginosa, Staphylococcus aureus) & & \\
\hline & & Antibacteriana & Inativo & richia coli) & & \\
\hline & Casca do caule & Antifúngica & Inativo & sporium sphaerospermum) & $k, m$ & 50 \\
\hline & & Citotóxica & Inativo & ia salina) & & \\
\hline B. verbascifolia & & Moluscicida & Ativo $(b$ & ialaria glabrata) & & \\
\hline & & & & Bacillus cereus, Pseudomonas aeruginosa, Staphylococcus aureus & $d$ & 50 \\
\hline & & Antibacteriana & Ativo & Bacillus subtilis, Staphylococcus aureus, Streptococcus faecalis & $k$ & 44 \\
\hline & & & Inativo & richia coli) & $d$ & 50 \\
\hline & & Antifíngica & & Cladosporium sphaerospermum & $d$ & 50 \\
\hline & & Antifúngica & Inativo & Candida albicans & $k$ & \\
\hline & Folha & Antimicobacteriana & Ativo $(N$ & acterium phlei) & $k$ & 44 \\
\hline & & Antimutagênica & Ativo $(L$ & hila melanogaster) & $m$ & 46 \\
\hline & & Antiviral & Ativo $(\mathrm{v}$ & herpes simples) & $k$ & 44 \\
\hline & & & & Artemia salina & $d, k$ & 50 \\
\hline & & Citotóxica & Inativo & Células Vero & $k$ & 44 \\
\hline & & Moluscicida & Ativo $(B$ & alaria glabrata) & $d$ & 50 \\
\hline
\end{tabular}

$a=$ extrato éter de petróleo, $b=$ extrato hexano, $\mathrm{c}=$ extrato diclorometano, $d=$ extrato diclorometano : metanol $(1: 1), e=$ extrato clorofórmio, $f=$ extrato acetato de etila, $g=$ extrato acetona, $h=$ extrato etanol, $i=$ extrato etanol a $10 \%, j=$ extrato etanol a $50 \%, k=$ extrato metanol, $l=$ extrato metanol a $80 \%, m=$ extrato aquoso, $n=$ extrato metanol : etanol : água : acido clorídrico $(69: 20: 10: 10: 1, \mathrm{v}: \mathrm{v}: \mathrm{v}: \mathrm{v}), o=$ substâncias obtidas do extrato clorofórmio, $p=$ substâncias obtidas do extrato metanol, $q=$ substâncias obtidas do extrato metanol a $80 \%, r=$ fração acetato de etila do extrato metanol, $s=$ fração aquosa do extrato metanol, $t=$ fração acetato de etila do extrato metanol a $80 \%$.

triterpênicos (estruturas de $\mathbf{3 4}$ a 56), além de ácido gálico e derivados (estruturas de 64 a 69), derivados do ácido quínico (estruturas de 70 a 73), aminoácidos (estruturas de $\mathbf{7 4}$ a 79) entre outras substâncias menos usuais (estruturas de $\mathbf{8 0}$ a $\mathbf{9 0}$ ) foram isolados de diferentes espécies do gênero Byrsonima. Mais detalhes sobre esses estudos são encontrados na Tabela 3 e na Figura 1 são encontradas as estruturas mencionadas acima e na referida tabela.

Em adição ao que foi informado na Tabela 3, a obtenção de algumas misturas semipurificadas de compostos pouco comuns no gênero Byrsonima também são relatadas na literatura. Das folhas de B. mi- 
Tabela 3. Substâncias isoladas de espécies do gênero Byrsonima (em ordem alfabética)

\begin{tabular}{|c|c|c|c|}
\hline Espécie & Parte estudada & Substâncias isoladas & Ref. \\
\hline B. basiloba & Folha & 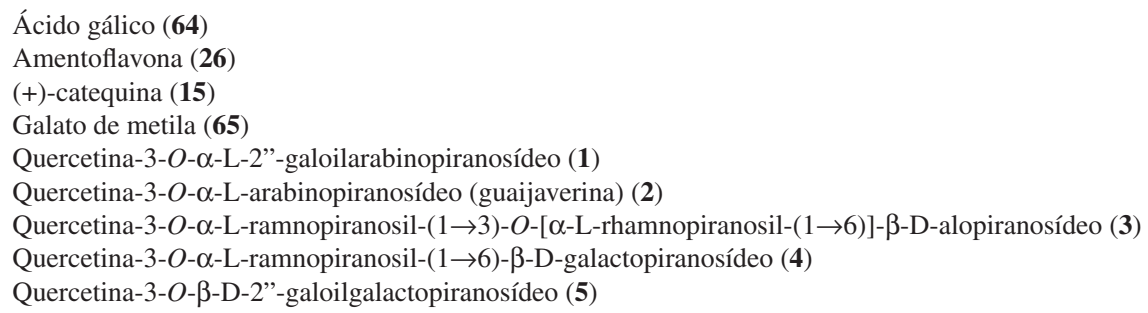 & $8,9,59$ \\
\hline B. bucidaefolia & Folha & $\begin{array}{l}\text { Galato de metila }(\mathbf{6 5}) \\
m \text {-trigalato de metila }(\mathbf{6 9})\end{array}$ & 10 \\
\hline
\end{tabular}

Ácido 3-O-galoil-quínico (70)

Ácido 3,4-O-digaloil-quínico (71)

Ácido 3,4,5-O-trigaloil-quínico (72)

Ácido 1,3,4,5-O-tetragaloil-quínico (73)

Amentoflavona (26)

(+)-catequina $(\mathbf{1 5})$

$(+)$-catequina-[ $4 \alpha \rightarrow 8]-(+)$-catequina $(\mathbf{1 6})$

$(+)$-catequina-[4 $\alpha \rightarrow 8]-(+)$-catequina-[4 $\alpha \rightarrow 8]-(+)$-catequina $(\mathbf{1 7})$

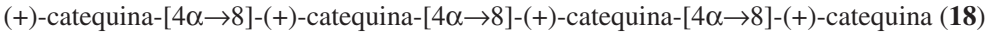

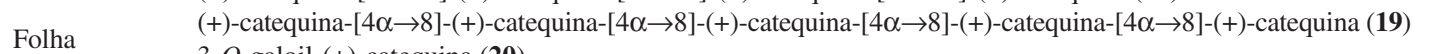

3-O-galoil-(+)-catequina (20)

B. crassa

3-O-galoil-(+)-catequina-[4 $\alpha \rightarrow 8]-3-O-$ galoil-(+)-catequina $(\mathbf{2 1})$

11,12 ,

13,15 ,

17,19

(-)-epicatequina (22)

Galato de (-)-epigalocatequina (23)

Galato de metila (65)

Quercetina (6)

Quercetina-3-O- $\alpha$-L-2"-galoilarabinopiranosídeo (1)

Quercetina-3- $O-\alpha$-L-arabinopiranosídeo (guaijaverina) (2)

Quercetina-3-O- $\beta$-D-2"-galoilgalactopiranosídeo (5)

Quercetina-3-O- $\beta$-D-galactopiranosídeo (hiperina) (7)

Casca do caule $\beta$-amirina(40)

Friedelina (39)

3-O-galoil-(+)-epicatequina (12)

3-O-galoil-(+)-epicatequina-[4 $\alpha \rightarrow 8]$-3-O-galoil-(+)-epicatequina (27)

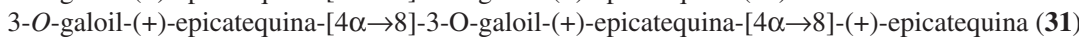

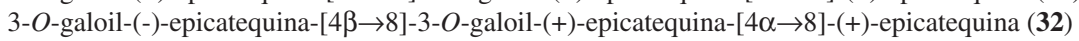

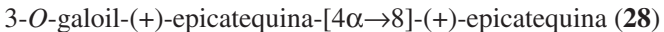

Casca do caule Ácido gálico (64)

$(+)$-catequina $(\mathbf{1 5})$

(+)-epicatequina (13)

$(+)$-epicatequina-[4 $\alpha \rightarrow 6]-(+)$-epicatequina $(\mathbf{3 3})$

$(+)$-epicatequina-[4 $\alpha \rightarrow 8]-(+)$-epicatequina $(\mathbf{2 9})$

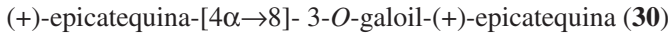

$\beta$-sitosterol (55)

1,2-di-O-(8-hexadecenoil)-3-O-( $\beta$-D-glicopiranosil)-glicerol (83)

1,2-di- $O$-(8-hexadecenoil)-3- $O$-(6-sulfo- $\alpha$-D-quinovopiranosil)-glicerol (80)

1,2-di- $O$-hexadecanoil-3- $O$-( $\beta$-D-glicopiranosil)-glicerol (84)

B. crassifolia

1,2 -di- $O$-tetradecanoil-3- $O$-(6-sulfo- $\alpha$-D-quinovopiranosil)-glicerol (81)

1,2-di-O-hexadecanoil-3-O-(6-sulfo- $\alpha$-D-quinovopiranosil)-glicerol (82)

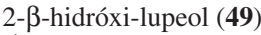

Ácido aspártico (77)

Ácido betulínico (50)

Ácido 2- $\alpha$-hidróxi-oleanólico (43)

Folha Ácido 2- $\beta$-hidróxi-oleanólico (44)

Ácido 5-hidróxi-pipecólico (74)

Ácido oleanólico (42)

Ácido pipecólico (75)

Betulina (51)

Betulinaldeído (52)

D-alanina (78)

Daucosterol (63)

Galato de metila $(\mathbf{6 5})$

Lupeol (48)

Quercetina (6) 
Tabela 3. continuação

\begin{tabular}{|c|c|c|c|}
\hline Espécie & Parte estudada & Substâncias isoladas & Ref. \\
\hline B. crassifolia & Folha & $\begin{array}{l}\text { Quercetina-3-O- } \alpha \text {-L-arabinopiranosídeo (guaijaverina) (2) } \\
\text { Quercetina-3-O- } \beta \text {-D-6"-galoilgalactopiranosídeo (8) } \\
\text { Quercetina-3-O- } \beta \text {-D-galactopiranosídeo (hiperina) (7) } \\
\text { Quercetina-3-O- } \beta \text {-D-glicopiranosídeo (isoquercetina) (9) } \\
\text { Prolina (76) } \\
\text { Ursenaldeído (37) } \\
\text { Valina (79) }\end{array}$ & $21,22,29$ \\
\hline B. fagifolia & Folha & 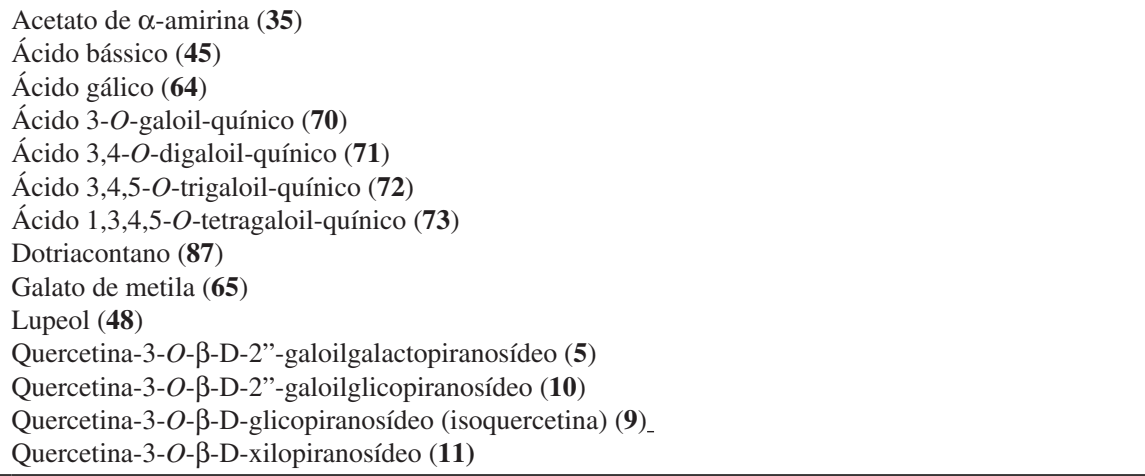 & $38,39,40$ \\
\hline \multirow[t]{3}{*}{ B. intermedia } & Folha & $\begin{array}{l}\text { Ácido gálico (64) } \\
\text { Amentoflavona (26) } \\
\text { Galato de metila (65) } \\
\text { Quercetina (6) } \\
\text { Quercetina-3-O- } \alpha \text {-L-2"-galoilarabinopiranosídeo (1) } \\
\text { Quercetina-3-O- } \alpha \text {-L-arabinopiranosídeo (guaijaverina) (2) } \\
\text { Quercetina-3-O- } \beta \text {-D-2"-galoilgalactopiranosídeo (5) } \\
\text { Quercetina-3-O- } \beta \text {-D-galactopiranosídeo (hiperina) (7) }\end{array}$ & 55 \\
\hline & Raiz & $\begin{array}{l}\text { B-amirina (40) } \\
\text { Ácido gálico }(\mathbf{6 4}) \\
\text { 1,2-benzenodiol (catecol, pirocatecol, pirocatequina) (66) } \\
\text { 1,2,3-benzenotriol (pirogalol, ácido pirogálico) }(\mathbf{6 7})\end{array}$ & 41,60 \\
\hline & Flor & Ácido (3R, 7R)-3,7-diacetóxi-docosanoico (ácido birsônico) (86) & 61 \\
\hline \multirow[b]{2}{*}{ B. microphylla } & Folha & $\begin{array}{l}\text { Galato de metila }(\mathbf{6 5}) \\
\text { Quercetina }(\mathbf{6})\end{array}$ & 62 \\
\hline & Madeira & 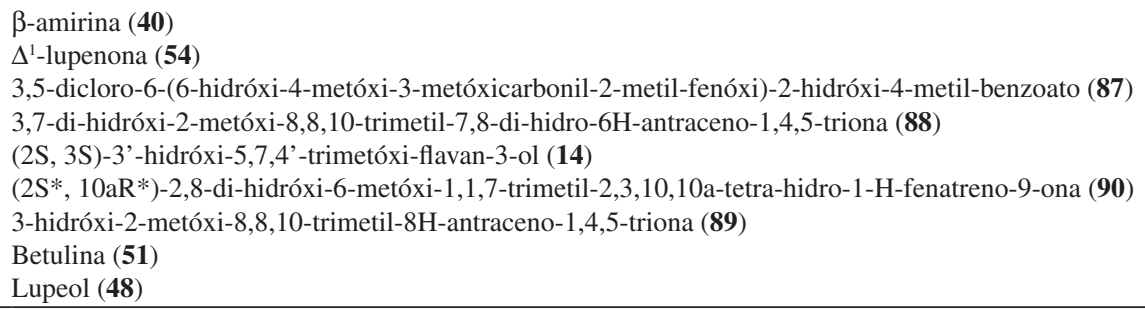 & 63,64 \\
\hline B. variabilis & Flor & $\begin{array}{l}\text { 3-O- } \beta \text {-D-glicopiranosil-3', 4', 5, } 7 \text { - tetra-hidroxiflavanona }(\mathbf{2 4}) \\
\text { 3', 4', 5, } 7 \text { - tetra-hidroxiflavanona }(\mathbf{2 5}) \\
\text { Galato de etila }(\mathbf{6 8})\end{array}$ & 65 \\
\hline \multirow{2}{*}{ B. verbascifolia } & Casca do caule & $\begin{array}{l}\beta \text {-amirina }(\mathbf{4 0}) \\
\beta \text {-amirirona }(\mathbf{4 7}) \\
\beta \text {-sitosterol }(\mathbf{5 5}) \\
\text { 3- } O \text {-acetil-lupeol }(\mathbf{5 3}) \\
\text { Ácido } 3 \text { - } O \text {-acetil-oleanólico }(\mathbf{4 6}) \\
\text { Friedelina }(\mathbf{3 9})\end{array}$ & 66 \\
\hline & Folha & $\begin{array}{l}\alpha \text {-amirina (34) } \\
\text { Ácido oleanólico (42) } \\
\text { Ácido ursólico (36) } \\
\text { Quercetina (6) } \\
\text { Quercetina-3-O- } \alpha \text {-L-arabinopiranosídeo (guaijaverina) (2) } \\
\text { Quercetina-3-O- } \beta \text {-D-glicopiranosídeo (isoquercetina) (9) }\end{array}$ & 43 \\
\hline
\end{tabular}

crophylla foi obtida uma mistura de seis triterpenos esterificados com ácidos graxos: 3b-eicosanato, -estearato e -palmitato de 24-hidróxiurs-12-enila (estruturas de $\mathbf{5 7}$ a 59, respectivamente) e 3b-eicosanato, -estearato e -palmitato de 24-hidróxi-olean-12-enila (estruturas de
60 a 62 , respectivamente). Nesse mesmo trabalho também foi obtida uma mistura de ácido oleanólico e ácido 3b, 2a-di-hidróxi-urs-12-en28-oico (estruturas 42 e $\mathbf{6 3}$, respectivamente). ${ }^{62}$ Outras misturas de triterpenos bem conhecidos foram obtidas das folhas de B. crassa $^{14}$ 
<smiles>Cc1cc(O)cc2c1CC(O)=C(c1ccc(O)c(O)c1)O2</smiles>

$\mathrm{OH}$

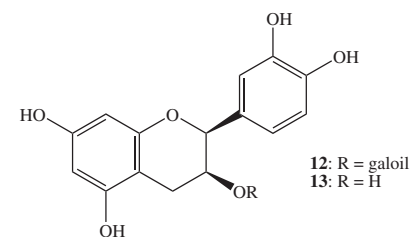

$\mathrm{OH}$<smiles>COc1cc(OC)c2c(c1)O[C@H](c1ccc(OC)c(O)c1)[C@H](O)C2</smiles><smiles>O=c1c(O)c(-c2ccc(O)c(O)c2)oc2cc(O)cc(O)c12</smiles>

24: $R_{1}=\beta$-D-glicopiranose

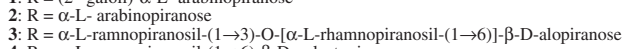
3: $R=\alpha$-L-ramnopiranosil-( $(1 \rightarrow 3)-O-[\alpha-L-$-rhamnopiras
: $R=\alpha$-L-ramnopiranosil- $(1 \rightarrow 6)-\beta$-D-galactopiranose $\begin{aligned} & \text { 4: } R=\alpha-L-\text {-ramnopiranosil- }(1 \rightarrow 6)-\beta-D- \\ & \text { 5: } R=(2 " \text {-galoil })-\beta \text {-D-galactopiranose }\end{aligned}$ 6: $R=H \quad H$-D-galactopiranose 8: $R=(6 "$-galoil) $-\beta$-D-galactopiranose 9: $R=\beta$-D-glicopiranose
10: $R=(2$-galoil)- $\beta$-D-glicopiranose
11: $R=\beta$-D-xilopiranosil<smiles>Cc1cc([C@@H]2Oc3cc(O)cc(O)c3[C@@H](C)[C@H](O)[C@H]2O)cc(O)c1O</smiles>

15: $R_{1}=R_{2}=R_{3}=R_{4}=H$

16: $\mathrm{R}_{1}=\mathrm{R}_{2}=\mathrm{H}, \mathrm{R}_{3}=\alpha-(+)$-catequina, $\mathrm{R}_{4}=\mathrm{H}$

17: $\mathrm{R}_{1}=\mathrm{R}_{2}=\mathrm{H}, \mathrm{R}_{3}=\alpha$-(+)-catequina- $[4 \alpha \rightarrow 8]$-(+)-catequina, $\mathrm{R}_{4}=\mathrm{H}$

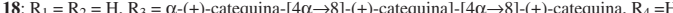

19: $R_{1}=R_{2}=H, R_{3}=\alpha-(+)$-catequina-[ $\left.4 \alpha \rightarrow 8\right]-(+)$-catequina]-[ $\left.4 \alpha \rightarrow 8\right]-(+)$-catequina-[ $\left.4 \alpha \rightarrow 8\right]-(+)$-catequina, $R_{4}=H$ 20: $\mathrm{R}_{1}=\mathrm{R}_{3}=\mathrm{R}_{4}=\mathrm{H}, \mathrm{R}_{2}=$ galoi

21: $\mathrm{R}_{1}=\mathrm{H}, \mathrm{R}_{2}=$ galoil, $\mathrm{R}_{3}=\alpha$-(+)-catequina-3- $O$-galoil

$\begin{array}{ll}\mathrm{OH} & \text { 22: } \mathrm{R}_{1}=\mathrm{OH}, \mathrm{R}_{2}=\mathrm{R}_{3}=\mathrm{H} \\ \mathrm{O} & \text { 23: } \mathrm{R}_{1}=\text { galoil, } \mathrm{R}_{2}=\mathrm{R}_{3}=\mathrm{H}, \mathrm{R}_{4}=\mathrm{OH}\end{array}$

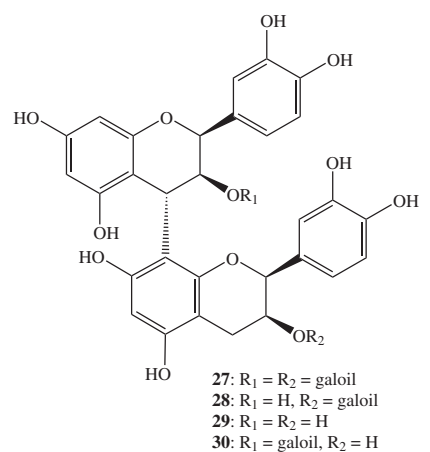

30: $R_{1}=$ galoil, $R_{2}=H$

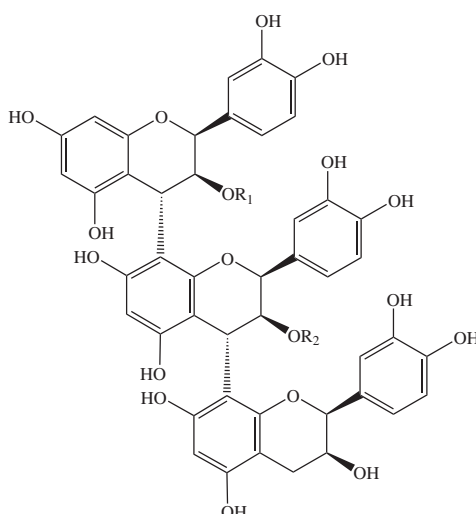

31: $\mathrm{R}=$ galoi

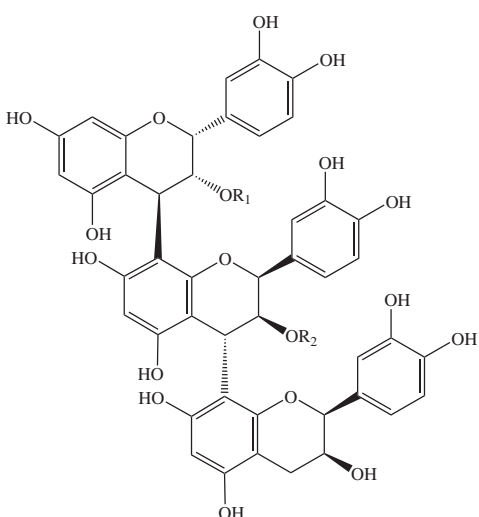

32: $\mathrm{R}=$ galoi<smiles>Oc1cc(O)c2c(c1)O[C@H](c1ccc(O)c(O)c1)[C@H](O)[C@H]2c1c(O)cc2c(c1O)C[C@@H](O)[C@H](c1ccc(O)c(O)c1)O2</smiles>

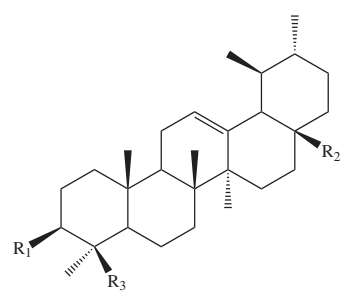

34: $\mathrm{R}_{1}=\mathrm{OH}, \mathrm{R}_{2}=\mathrm{CH}_{3}$

35: $\mathrm{R}_{1}=\mathrm{OCOCH}_{3}, \mathrm{R}_{2}=\mathrm{CH}_{3}$

36: $\mathrm{R}_{1}=\mathrm{OH}, \mathrm{R}_{2}=\mathrm{COOH}$

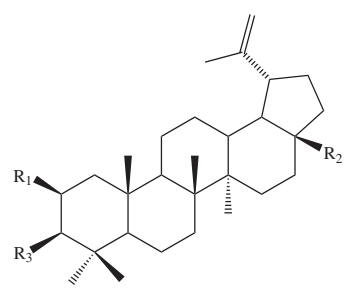

48: $\mathrm{R}_{1}=\mathrm{H}, \mathrm{R}_{2}=\mathrm{CH}_{3}, \mathrm{R}_{3}=\mathrm{OH}$ 49: $\mathrm{R}_{1}=\mathrm{OH}, \mathrm{R}_{2}=\mathrm{CH}_{3}, \mathrm{R}_{3}=\mathrm{OH}$ 50. $\mathrm{R}_{1}=\mathrm{OH}, \mathrm{R}_{2}=\mathrm{COOH}, \mathrm{R}_{3}=\mathrm{OH}$ 51: $\mathrm{R}_{1}=\mathrm{H}_{1} \mathrm{R}_{2}=\mathrm{CH}_{2} \mathrm{OH}, \mathrm{R}_{3}=\mathrm{OH}$ 52: $\mathrm{R}_{1}=\mathrm{OH}, \mathrm{R}_{2}=\mathrm{CHO}, \mathrm{R}_{3}=\mathrm{OH}$

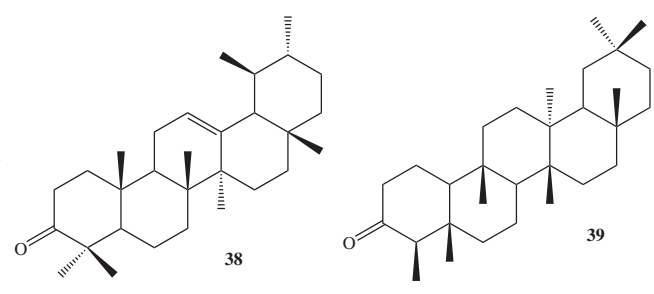<smiles>C=C(C)C1CCC2(C)CCC3C(CCC4C3CCC3C(C)(C)C(=O)C=CC43C)C12</smiles><smiles>CCC#CC1CC(CCC(CC)C(C)C)C2CC=C3C[C@@H](C)CCC3(C)C1CCC2C</smiles>

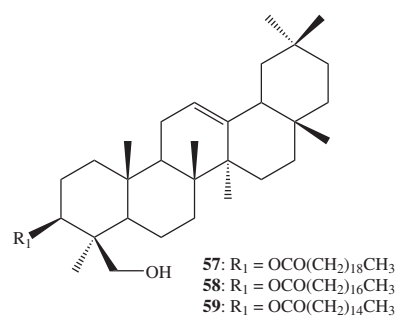<smiles>O=C1c2c(O)cc(O)cc2O[C@@H](c2ccc(O)c(O)c2)[C@@H]1O</smiles><smiles>O=c1cc(-c2ccc(O)c(-c3c(O)cc(O)c4c(=O)cc(-c5ccc(O)cc5)oc34)c2)oc2cc(O)cc(O)c12</smiles>

Figura 1. Estruturas das substâncias obtidas de espécies do gênero Byrsonima 
<smiles>[R]c1cc(Br)cc(O)c1O</smiles>

64: $\mathrm{R}_{1}=\mathrm{COOH}, \mathrm{R}_{2}=\mathrm{OH}$ 65: $\mathrm{R}_{1}=\mathrm{COOCH}_{3}, \mathrm{R}_{2}=\mathrm{OH}$ 66: $\mathrm{R}_{1}=\mathrm{R}_{2}=\mathrm{H}$

67: $\mathrm{R}_{1}=\mathrm{H}, \mathrm{R}_{2}=\mathrm{OH}$

68: $\mathrm{COOC}_{2} \mathrm{H}_{5}, \mathrm{R}_{2}=\mathrm{OH}$<smiles>O=C(Oc1cc(O)c(O)c(OC(=O)c2cc(O)cc(O)c2O)c1)c1cc(O)c(O)c(O)c1</smiles>

DERIVADOS DO ÁCIDO QUÍNICO

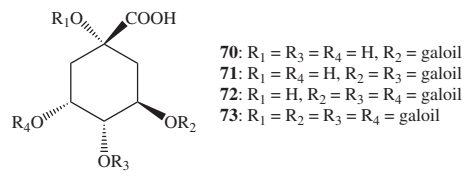

\section{AMINOÁCIDOS}
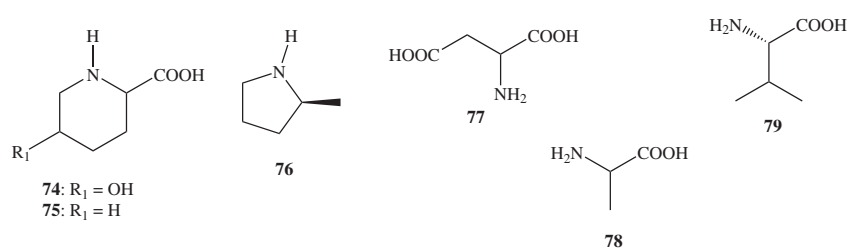

OUTRAS SUBSTÂNCIAS<smiles>[R]OC[C@H]([2H])COC(OCC(O)C(O)C(O)CO)C(O)CO</smiles>

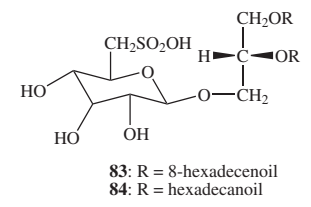<smiles>CCCCC[C@H](CCC[C@H](CC(=O)O)OC(C)=O)OC(C)=O</smiles><smiles>COC1=C(O)C(=O)c2c(cc3c(c2C)C(=O)CC(O)C3(C)C)C1=O</smiles>
88<smiles>COC1=C(O)C(=O)c2c(cc3c(c2C)C(=O)C=CC3(C)C)C1=O</smiles>

89<smiles>COc1cc2c(c(O)c1C)C(=O)CC1C2=CC[C@@H](O)C1(C)C</smiles>

Figura 1. Estruturas das substâncias obtidas de espécies do gênero Byrsonima (continuação)

e B. fagifolia, ${ }^{39}$ e misturas de (+)-catequina e (-)-epicatequina foram obtidas das folhas de B. crassifolia $^{22}$ e B. intermedia. ${ }^{55}$

Cerca de 50 compostos voláteis entre alcoóis, cetonas, aldeídos e ésteres comuns foram identificados nos frutos de B. crassifolia por cromatografia gasosa acoplada à espectrometria de massas. ${ }^{67,68}$ Diasteroisômeros de catequina foram identificados e quantificados nas folhas de B. basiloba, B. crassa, B. intermedia e B. verbascifolia por cromatografia líquida de alta eficiência (CLAE) ${ }^{69}$ Nas folhas de $B$. crispa foi detectada a presença de constituintes cianogênicos por meio de reações físico-químicas, porém, nenhum composto foi identificado. ${ }^{70}$ Cinco diferentes heterosídeos de quercetina foram identificados nas folhas de B. fagifolia por CLAE, no entanto, os açúcares presentes não puderam ser determinados. ${ }^{40}$

\section{CONSIDERAÇÕES FINAIS}

Os resultados apresentados nesta revisão, referentes a 40 anos de pesquisas com este gênero, estão restritos a 13 das suas 150 espécies. A espécie com maior número de trabalhos, tanto com enfoque fitoquímico quanto farmacológico, é $B$. crassifolia, que é amplamente distribuída nas Américas Central e do Sul e tem amplo uso medicinal em toda essa região. Apenas estudos farmacológicos foram realizados com B. coccolobifolia, B. gardneriana, B. japurensis e B. sericea. Por outro lado, apesar de alguns dos constituintes de $B$. microphylla e $B$. variabilis já serem conhecidos, as mesmas ainda não foram investigadas farmacologicamente.

A maior parte dos estudos não teve continuidade ou ainda não se conhece o metabólito responsável pela atividade apresentada. Apenas cinco trabalhos apresentados nesta revisão investigaram as atividades biológicas de compostos isolados das espécies, ${ }^{14,15,18,20,22,39}$ detendo-se, a maioria, em avaliar atividades farmacológicas de extratos brutos, predominando a investigação de extratos polares das folhas, com destaque para extratos metanólicos.

A forte presença de flavonoides e terpenos nas espécies desse gênero sugere que elas podem apresentar atividades anti-inflama- tória, anti-hiperlipidêmica e antitumoral. ${ }^{71}$ Entretanto, apenas um trabalho investigou a atividade anti-inflamatória tópica da espécie B. crassifolia $^{34} \mathrm{e}$ outro trabalho recente investigou o potencial antihiperlipidêmico e antiglicemiante dos frutos e sementes da mesma espécie $^{53}$ e um terceiro trabalho avaliou a atividade anti-inflamatória e antiproliferativa de $B$. crassa. ${ }^{20}$

É interessante destacar a baixa toxicidade dessas espécies, evidenciada pelos resultados dos trabalhos em que foram investigados seus efeitos tóxicos, citotóxicos, genotóxicos ou mutagênicos, in vivo e in vitro. Apenas B. crassa e B. intermedia apresentaram atividade mutagênica sobre culturas de Salmonella typhimurium ${ }^{11,55}$ e $B$. sericea foi tóxica a camudongos e peixes. ${ }^{57} \mathrm{Tal}$ fato sugere relativa segurança para o avanço das pesquisas acerca do potencial farmacológico dessas espécies, que têm ampla representatividade e uso medicinal popular no Brasil.

\section{AGRADECIMENTOS}

Ao Conselho Nacional de Desenvolvimento Científico e Tecnológico $(\mathrm{CNPq})$ e à Fundação Coordenação de Aperfeiçoamento de Pessoal de Nível Superior (CAPES).

\section{REFERÊNCIAS}

1. Davis, C. C.; Bell, C. D.; Mathews, S.; Donoghue, M. J.; P. Natl. Acad. Sci. U.S.A. 2002, 99, 6833; Lombello, R. A.; Forni-Martins; E. R.; Acta Bot. Bras. 2003, 17, 171.

2. Gable, R. S.; Addiction 2007, 102, 24; McKenna, D. J.; Pharmacol. Therapeut. 2004, 102, 111 ; Riba, J.; Valle, M.; Urbano, G.; Yritia, M.; Morte, A.; Barbanoj, M. J.; J. Pharm. Exp. Ther. 2003, 306, 73; Schwars, M. J.; Houghton, P. J.; Rose, S.; Jenner, P.; Lees, A. D.; Pharmacol., Biochem. Behav. 2003, 75, 627; Aguilar-Santamaría, L.; Ramírez, G.; Herrera-Arellano, A.; Zamilpa, A.; Jiménez, J. E.; Alonso-Cortés, D.; Cortés-Gutiérrez, E. I.; Ledesma, N.;Tortoriello, J.; J. Ethnopharmacol. 2007, 109, 35; Herrera-Ruiz, M.; Jiménez- 
Ferrer, J. E.; De Lima, T. C. M.; Avilés-Montes, D.; Pérez-García, D.; González-Cortazar, M.; Tortoriello, J.; Phytomedicine 2006, 13, 23; Taketa, A. T. C.; Lozada-Lechuga, J.; Fragoso-Serrano, M.; Villareal, M. L.; Pereda-Miranda, R.; J. Nat. Prod. 2004, 67, 644; Roman-Júnior, W. A.; Cardoso, M. L. C.; Vilegas, W.; Nakamura, C. V.; Dias-Filho, B. P.; Mello, J. C. P.; Acta Farm. Bonaer. 2005, 24, 543; Melo, F. L.; Benati, F. J.; Roman-Júnior, W. A.; Mello, J. C. P.; Nozawa, C.; Linhares, R. E. C.; Microbiol. Res. 2008, 163, 136; Galvão, S. M. P.; Marques, L. C.; Oliveira, M. G. M.; Carlini, E. A.; J.Ethnopharmacol. 2002, 79, 305.

3. Dias, S. M. C.; Arq. Inst. Biol. 1977, 44, 15.

4. Teixeira, L. A. G.; Machado, I. C.; Acta Bot. Bras. 2000, 14, 347.

5. Cardoso, C. R. P.; Dissertação de Mestrado, Universidade Estadual Paulista, Brasil, 2006.

6. Vicentini, A.; Anderson, W. R. Em Flora da Reserva Ducke - guia de identificação das plantas vasculares de uma floresta de terra-firme da Amazônia Central; Ribeiro, J. E. L. S.; Hopkins, M. J. G.; Vicentini, A.; Sothers, C. A.; Costa, M. A. S.; Brito, J. M.; Souza, M. A.; Martins, L. H. P.; Lohmann, L. G.; Assunção, P. A. C. L.; Pereira, E. C.; Silva, C. F.; Mesquita, M. R.; Procópio, L. C., eds.; INPA-DFID: Manaus, 1999, p. 505-511.

7. Vallilo, M. I.; Pastore, J. A.; Eston, M. R.; Garbelotti, M. L.; Oliveira, E.; Rev. Inst. Flor. 2007, 19, 39.

8. Lira, W. M.; Santos, F. V.; Sannomiya, M.; Rodrigues, C. M.; Vilegas, W.; Varanda, E. A.; J. Med. Food 2008, 11, 111.

9. Figueiredo, M. E.; Michelin, D. C.; Sannomiya, M.; Silva, M. A.; Santos, L. C.; Almeida, L. F. R.; Salgado, H. R. N.; Vilegas, W.; Rev. Bras. Cienc. Farm. 2005, 41, 79.

10. Castillo-Avila, G. M.; García-Sosa, K.; Peña-Rodríguez, L. M.; Nat. Prod. Commun. 2009, 4, 83.

11. Cardoso, C. R. P.; Cólus, I. M. S.; Bernardi, C. C.; Sannomiya, M.; Vilegas, W.; Varanda, E. A.; Toxicology 2006, 225, 55.

12. Sannomiya, M.; Rodrigues, C. M.; Coelho, R. G.; Santos, L. C.; Hiruma-Lima, C. A.; Brito, A. R. M. S.; Vilegas, W.; J. Chromatogr., A 2004, 1035, 47.

13. Sannomiya, M.; Michelin, D. C.; Rodrigues, C. M.; Santos, L. C.; Salgado, H. R. N.; Hiruma-Lima, C. A.; Brito, A. R. S. M.; Vilegas, W.; Rev. Bras. Cienc. Farm. Bás. Aplic. 2005, 26, 71.

14. Higuchi, C. T.; Pavan, F. R.; Leite, C. Q. F.; Sannomiya, M.; Vilegas, W.; Leite, S. R. A.; Sacramento, L. V. S.; Sato, D. N.; Quim. Nova. 2008, 31, 1719.

15. Rolim de Almeida, L. F.; Sannomiya, M.; Rodrigues, C. M.; Delachive, M. E.; Santos, L. C.; Vilegas, W.; Feo, V.; J. Plant. Interact. 2007, 2, 121.

16. Bonacorsi, C.; Raddi, M. S. G.; Carlos, I. Z.; Sannomiya, M.; Vilegas W.; BMC Complementary and Alternative Medicine (online) 2009, 9, 1.

17. Sannomiya, M.; Fonseca, V. B.; Silva, M. A.; Rocha, L. R.; Santos, L. C.; Hiruma-Lima, C. A.; Souza Brito, A. R.; Vilegas, W.; J. Ethnopharmacol. 2005, 97, 1.

18. Nishijima, C. M.; Rodrigues, C. M.; Silva, M. A.; Lopes-Ferreira, M.; Vilegas, W.; Hiruma-Lima, C. A.; Molecules 2009, 14, 1072.

19. Sannomiya, M.; Montoro, P.; Piacente, S.; Pizza, C.; Brito, A. R.; Vilegas, W.; Rapid Commun. Mass Spectrom. 2005, 19, 2244.

20. Carli, C. B. A.; Matos, D. C.; Lopes, F. C. M.; Maia, D. C. G.; Dias, M. B.; Sannomiya, M.; Rodrigues, C. M.; Andreo, M. A.; Vilegas, W.; Colombo, L. L.; Carlos, I. Z.; Z. Natuforsch. C 2009, 64, 32.

21. Amarquaye, A.; Che, C.; Bejar, E.; Malone, M. H.; Fong, H. H. S.; Planta Med. 1994, 60, 85.

22. Bejar, E.; Amarquaye, A.; Che, C.; Malone, M. H.; Fong, H. H. S.; Int. J. Pharmacogn. 1995, 33, 25.

23. Berger, I.; Barrientos, A. C.; Cáceres, A.; Hernández, M.; Rastrelli, L.; Passreiter, C. M.; Kubelka, W.; J. Ethnopharmacol. 1998, 62, 107.

24. Peraza-Sánchez, S. R.; Poot-Kantún, S.; Torres-Tapia, L. W.; May-Pat, F.; Simá-Polanco, P.; Cedillo-Rivera, R.; Pharmaceut. Biol. 2005, 43, 594.
25. Cáceres, A.; López, B. R.; Giron, M. A.; Logemann, H.; J. Ethnopharmacol. 1991, 31, 263.

26. Cáceres, A.; López, B. R.; Juárez, X.; Aguila, J.; García, S.; J. Ethnopharmacol. 1993, 40, 207.

27. Cáceres, A.; López, B.; González, S.; Berger, I.; Tada, I.; Maki, J.; J. Ethnopharmacol. 1998, 62, 195.

28. Geiss, F.; Heinrich, M.; Hunkler, D.; Rimpler, H.; Phytochemistry 1995 , 39,635 .

29. Rastrelli, L.; De Tommasi, N.; Berger, I.; Caceres, A.; Saravia, A.; De Simone, F.; Phytochemistry 1997, 45, 647.

30. Silva, E. M.; Souza, J. N. S.; Rogez, H.; Rees, J. F.; Larondelle, Y.; Food Chem. 2007, 101, 1012.

31. Martinez-Vazquez, M.; González-Esquinca, A. R.; Luna, L. C.; Gutiérrez, M. N. M.; García-Argáez, A. N.; J. Ethnopharmacol. 1999, 66, 79.

32. Cáceres, A.; Figueroa, L.; Taracena, A. M.; Samayoa, B.; J. Ethnopharmacol. 1993, 39, 77.

33. Cáceres, A.; Fletes, L.; Aguilar, L.; Ramirez, O.; Figueroa, L.; Taracena, A. M.; Samayoa, B.; J. Ethnopharmacol. 1993, 38, 31.

34. Maldini, M.; Sosa, S.; Montoro, P.; Giangaspero, A.; Ballick, M. J.; Pizza, C.; Della Loggia, R.; J. Ethnopharmacol. 2009, 122, 430.

35. Cáceres, A.; Jauregui, E.; Herrera, D.; Logemann, H.; J. Ethnopharmacol. 1991, 33, 277.

36. Cáceres, A.; Cano, O.; Samayoa, B.; Aguilar, L.; J. Ethnopharmacol. 1990, 30, 55 .

37. Bejar, E.; Malone, M. H.; J. Ethnopharmacol. 1993, 39, 141.

38. Lima, Z. P.; Santos, R. C.; Torres, T. U.; Sannomiya, M.; Rodrigues, C. M.; Santos, L. C.; Pellizzon, C. H.; Rocha, L. R. M.; Vilegas, W.; Brito, A. R. M. S.; Cardoso, C. R. P.; Varanda, E. A.; Moraes, H. P.; Bauab, T. M.; Carli, C.; Carlos, I. Z.; Hiruma-Lima, C. A.; J. Ethnopharmacol. 2008, 120, 149.

39. Higuchi, C. T.; Sannomiya, M.; Pavan, F. R.; Leite, S. R. A.; Sato, D. N.; Franzblau, S. G.; Sacramento, L. V. S.; Vilegas, W.; Leite, C. Q. F.; eCAM. 2008, 1 .

40. Sannomiya, M.; Santos, L. C.; Carbone, V.; Napolitano, A.; Piacente, S.; Pizza, C.; Souza-Brito, A. R. M.; Vilegas, W.; Rapid Commun. Mass Spectrom. 2007, 21, 1393.

41. Silva, J. B.; Rev. Farm. Bioquim. Univ. São Paulo 1970, 8, 187.

42. Boscolo, O. H.; Mendonça-Filho, R. F. W.; Meneses, F. S.; Senna-Valle, L.; Rev. Bras. Plantas Med. 2007, 9, 8.

43. Dosseh, C.; Morreti, C.; Tessier, A. M.; Delaveau, P.; Plantes Médicinales et Phytothérapie 1980, 14, 136.

44. Lopez, A.; Hudson, J. B.; Towers, G. H. N.; J. Ethnopharmacol. 2001 , 77, 189.

45. Delaveau, P.; Lallouette, P.; Tessier, A. M.; Planta Med. 1980, 40, 49.

46. Mendanha, D. M.; Ferreira, H. D.; Felício, L. P.; Silva, E. M.; Pereira, D. G.; Nunes, W. B.; Carvalho, S.; Genet. Mol. Res. 2010, 9, 69.

47. Michelin, D. C.; Sannomiya, M.; Figueiredo, M. E.; Rinaldo, D.; Santos, L. C.; Souza-Brito, A. R. M.; Vilegas, W.; Salgado, H. R. N.; Rev. Bras. Farmacog. 2008, 18 (supl.), 690.

48. Arantes, V. P.; Sato, D. N.; Vilegas, W.; Santos, L. C.; Leite, C. Q. F.; Rev. Bras. Cienc. Farm. Bás. Aplic. 2005, 26, 195.

49. Peraza-Sánchez, S. R.; Cen-Pacheco, F.; Noh-Chimal, A.; May-Pat, F.; Simá-Polanco, P.; Dumonteil, E.; García-Miss, M. R.; Mut-Martín, M.; Fitoterapia 2007, 78, 315.

50. Alves, T. M. A.; Silva, A. F.; Brandão, M.; Grandi, T. S. M.; Smânia, E. F. A.; Smânia-Junior, A.; Zani, C. L.; Mem. Inst. Oswaldo Cruz, 2000, $95,367$.

51. Leite, C. F. Q.; Sato, D. N.; Higuchi, C. T.; Sannomiya, M.; Pavan, F. R.; Vilegas, W.; Rev. Bras. Pl. Med. 2008, 10, 63.

52. Cifuentes, C. M.; Gómez-Serranillos, M. P.; Iglesias, I.; Fresno, A. M. V.; J. Ethnopharmacol. 2001, 1, 223.

53. Perez-Gutierrez, R. M.; Muñiz-Ramirez, A.; Gomez; Y. G.; Ramírez, E. B.; Plant Food Hum. Nutr. 2010, 65, 350. 
54. David, J. P.; Meira, M.; David, J. M.; Brandão, H. N.; Branco, A.; Fatima, A. M.; Barbosa, M. R.; Queiroz, L. P.; Giulietti, A. M.; Fitoterapia 2007, 78, 215.

55. Sannomiya, M.; Cardoso, C. R. P.; Figueiredo, M. E.; Rodrigues, C. M.; Santos, L. C.; Santos, F. V.; Serpeloni, J. M.; Colus, I. M.; Vilegas, W.; Varanda, E. A.; J. Ethnopharmacol. 2007, 112, 319.

56. Graham, J. G.; Pendland, S. L.; Prause, J. L.; Danzinger, L. H.; Vigo, S.; Cabieses, F.; Farnsworth, N. R.; Phytomedicine 2003, 10, 528.

57. Barros, G. S. G.; Matos, F. J. A.; Vieira, J. E. V.; Sousa, M. P.; Medeiros, M. C.; J. Pharm. Pharmacol. 1970, 22, 116.

58. Silva, M. J. M.; Sousa, M. P.; Rouquayrol, M. Z.; Rev. Bras. Farm. 1971, Ano LII, 117.

59. Sannomiya, M.; Figueiredo, M. E.; Silva, M. A.; Rodrigues, C. M.; Santos, L. C.; Souza-Brito, A. R.; Vilegas, W.; Nat. Prod. Commun. 2007, 2, 829 .

60. Silva, J. B.; Rev. Farm. Bioquim. Univ. São Paulo. 1970, 8, 53.

61. Reis, M. G.; Faria, A. D.; Santos, I. A.; Amaral, M. C. E.; Marsaioli, A. J.; J. Chem. Ecol. 2007, 33, 1421.
62. Mendes, C. C.; Cruz, F. G.; David, J. M.; Nascimento, I. P.; David, J. P.; Quim. Nova 1999, 22, 185.

63. Aguiar, R. M.; David, J. P.; David, J. M.; Phytochemistry 2005, 66, 2388.

64. Rocha, J. H.; Cardoso, M. P.; David, J. P.; David, J. M.; Biosci., Biotechnol., Biochem. 2006, 70, 2759.

65. Aragão, P. C. A.; Toledo, J. B.; Morais, A. A.; Braz-Filho, R. Quim. Nova 1990, 13, 254.

66. Gottlieb, O. R.; Mendes, P. H.; Magalhães, M. T.; Phytochemistry 1975, $14,1456$.

67. Rezende, C. M.; Fraga, S. R. G.; J. Braz. Chem. Soc. 2003, 14, 425

68. Alves, G. L.; Franco, M. R. B.; J. Chromatogr., A 2003, 985, 297.

69. Rinaldo, D.; Batista-Junior, J. M.; Rodrigues, J.; Benfatti, A. C.; Rodrigues, C. M.; Santos, L. C.; Furlan, M.; Vilegas, W.; Chirality 2010, $22,726$.

70. Thomsen, K.; Brimer, L.; Bot. J. Linn. Soc. 1997, 124, 273.

71. Harborne, J. B.; Williams, C. A.; Phytochemistry 2000, 55, 481; Liu, J.; J. Ethnopharmacol. 1995, 49, 57. 